\title{
A Generalized Allosteric Mechanism for cis-Regulated Cyclic Nucleotide Binding Domains
}

\author{
Alexandr P. Kornev' ${ }^{1}$, Susan S. Taylor ${ }^{2,3 *}$, Lynn F. Ten Eyck ${ }^{1,2}$ \\ 1 San Diego Supercomputer Center, University of California San Diego, La Jolla, California, United States of America, 2 Department of Chemistry and Biochemistry, \\ University of California San Diego, La Jolla, California, United States of America, 3 Howard Hughes Medical Institute, University of California San Diego, La Jolla, California, \\ United States of America
}

\begin{abstract}
Cyclic nucleotides (cAMP and cGMP) regulate multiple intracellular processes and are thus of a great general interest for molecular and structural biologists. To study the allosteric mechanism of different cyclic nucleotide binding (CNB) domains, we compared cAMP-bound and CAMP-free structures (PKA, Epac, and two ionic channels) using a new bioinformatics method: local spatial pattern alignment. Our analysis highlights four major conserved structural motifs: 1) the phosphate binding cassette (PBC), which binds the CAMP ribose-phosphate, 2) the "hinge," a flexible helix, which contacts the PBC, 3) the $\beta_{2,3}$ loop, which provides precise positioning of an invariant arginine from the $\mathrm{PBC}$, and 4) a conserved structural element consisting of an $\mathrm{N}$ terminal helix, an eight residue loop and the A-helix (N3A-motif). The PBC and the hinge were included in the previously reported allosteric model, whereas the definition of the $\beta_{2,3}$ loop and the N3A-motif as conserved elements is novel. The N3Amotif is found in all cis-regulated CNB domains, and we present a model for an allosteric mechanism in these domains. Catabolite gene activator protein (CAP) represents a trans-regulated CNB domain family: it does not contain the N3A-motif, and its long range allosteric interactions are substantially different from the cis-regulated CNB domains.
\end{abstract}

Citation: Kornev AP, Taylor SS, Ten Eyck LF (2008) A Generalized Allosteric Mechanism for cis-Regulated Cyclic Nucleotide Binding Domains. PLoS Comput Biol 4(4): e1000056. doi:10.1371/journal.pcbi.1000056

Editor: Rama Ranganathan, HHMI/UT Southwestern Medical Center, United States of America

Received August 22, 2007; Accepted March 11, 2008; Published April 11, 2008

Copyright: (c) 2008 Kornev et al. This is an open-access article distributed under the terms of the Creative Commons Attribution License, which permits unrestricted use, distribution, and reproduction in any medium, provided the original author and source are credited.

Funding: This work was supported by National Science Foundation grant NSF-DBI 99111196, National Institute of General Medical Sciences grant GM70996 (to LFTE), National Institutes of Health grant GM19301, and National Science Foundation grant DBI0217951 (to SST).

Competing Interests: The authors have declared that no competing interests exist.

*E-mail: staylor@ucsd.edu

\section{Introduction}

Cyclic adenosine monophosphate (cAMP) is an important second messenger, which regulates a large variety of cellular processes, including metabolism, cell shape transformation, gene transcription, photoreception and chemosensation [1-5]. All cAMP-binding proteins in both pro- and eukaryotes share a small module - the cyclic nucleotide binding domain (CNB domain), which is typically fused to another domain. The GNB domain contains a contiguous $\beta$ subdomain and a non-contiguous $\alpha$-subdomain (Figure 1). The former is a relatively rigid eight-stranded $\beta$-sandwich, which accommodates the cyclic nucleotide molecule. The flexible helical $\alpha$-subdomain can accept substantially different configurations, which translates the allosteric signal [6]. Recent structure studies of cAMPdependent protein kinase (PKA) demonstrated, that CNB domains toggle between two stable conformations: bound to cAMP (so called B-form [7]), or to catalytic subunit of PKA (H-form) [8,9]. The intermediate, non bound form (apo-form) is characterized by high backbone flexibility [10-12] and is apparently represented by a dynamic ensemble of multiple configurations.

The $\beta$-subdomain contains a highly conserved element, the Phosphate Binding Cassette (PBC), which is 14-residues long and contains a short flexible $\alpha$-helix (B'-helix). Ribose-phosphate of cAMP, protected by the $\beta$-sandwich from the outside solution, forms six strong hydrogen bonds to the PBC. Due to these interactions, the B'-helix moves towards the cAMP molecule and adopts a compact conformation (Figure 1). Such movement causes a substantial rearrangement of the $\alpha$-subdomain both in its $\mathrm{N}$ - and
C-terminal parts. The latter contains the so called "hinge" [13], which consists of two consecutive $\alpha$-helices (B and $\mathrm{C}$ ). These helices are remarkably flexible and due to a strong connection to the B'-helix perform a swing-like motion: moving towards cAMP in the B-form (Figure 1A). The $\mathrm{N}$-terminal part of the $\alpha$ subdomain, which typically consists of two (short) helices and was called the "N-terminal helical bundle" [14], moves in an opposite way; in the B-form it moves away from the PBC, facilitating the hinge closure. In the $\mathrm{H}$-form the $\mathrm{N}$-terminal helices move towards the cAMP and make a contact to the B'-helix, filling the void space that results from the hinge opening (Figure 1B).

The other part of the cAMP molecule, the adenine ring, acts as a hydrophobic moiety, which stacks against a "capping residue" in all known CNB domain structures [15] (Figure 1A). Mutation studies have established the importance of this contact for stabilization of the B-form and cooperative cAMP-induced activation of the PKA holoenzyme [8].

A recent review summarizes this information into a general model for the CNB domain allosteric mechanism for PKA, Rap guanine nucleotide-exchange factor (Epac) and hyperpolarizationactivated cyclic-nucleotide-modulated channel (HCN) [14]. Although this model is in a good correspondence with much experimental data, two important issues remained unclear. First, as the authors mentioned, the N-terminal helical bundle is replaced by a single helix in the catabolite gene activator protein (CAP). This raises a question about the role of the helical bundle and its functional and structural conservation. Is it a universal part of the CNB domain or it is a part of protein-protein interface 


\section{Author Summary}

Cyclic nucleotides are small regulatory molecules which transmit signal from receptors positioned on a living cell membrane into the cell interior and regulate multiple biological processes ranging from bacteria to humans. Such regulation occurs through binding of the cyclic nucleotides to the corresponding proteins. All such proteins contain a relatively small domain responsible for the cyclic nucleotide binding. The most important task is to understand how cyclic nucleotide binding (CNB) domains translate the signal into a biological response. In this work, we studied changes in different CNB domains induced by the cyclic nucleotides using a new method for comparison of protein structures: local spatial patterns alignment. This novel method compares protein molecules and detects conserved spatial patterns comprised of similar amino acid residues. Moreover, it ranks the detected residues with respect to their functional or structural importance. Our results show that there are at least two different families of CNB domains. The first family has four structural elements which perform the signal translation. Two of these elements were known previously and two are novel. The second family can translate the signal using only three elements, but they have to work in pairs to provide interaction between the functional elements.

between the CNB domain and the host protein? The second problem is related to the loop located between $\beta_{2}$ and $\beta_{3}$ strands. A series of publications demonstrated, that it is an important element of allosteric mechanism in the PKA RI $\alpha$ :A-domain [1618], but it was not included in the model described by Rehmann et al [14] and was not considered to be a universal element.

To elucidate the cAMP induced allosteric mechanism that is conserved in different CNB domains, we used a recently developed method for protein structure comparison: Local Spatial Patterns (LSP) alignment that is capable of detecting similar patterns made up by amino acid residues in space. It is fast and does not require preliminary sequence or structural alignment of the compared proteins. Earlier we used it for comparison of protein surfaces of several CNB domains in the B-form and detected a conserved set of hydrophobic residues protecting the cAMP ribose-phosphate [15]. Here we considered both water accessible and buried residues of both B- and $\mathrm{H}$-forms of four different CNB domains: PKA, HCN, Epac and bacterial cyclic nucleotide modulated potassium channel (MloK1) [19]. The recently reported structures of two PKA holoenzymes [8,20] have allowed us for the first time to analyze both conformational states of multiple CNB domains.

Our analysis has shown that there are four elements conserved in all known CNB domains, with the exception of the CAP: the $\mathrm{PBC}$, the hinge, the $\beta_{2,3}$-loop and the "N3A-motif". The latter consists of the A-helix, a preceding eight residue loop and a short N-terminal helix. The loop contains a set of $310^{\text {-turns, and is }}$ termed "the $33_{10}$-loop". Based on these results, we propose a general model for the allosteric mechanism in CNB domains, which we called cis-regulated domains. In CAP the N3A-motif is reduced to a single A-helix. The difference between CAP and other CNB domains is discussed.

\section{Results}

\section{LSP Alignment Defines CAMP-Induced Conformational Changes in CNB Domains}

The LSP alignment is a new method to compare protein molecules. It is based on a graph-theoretical representation of
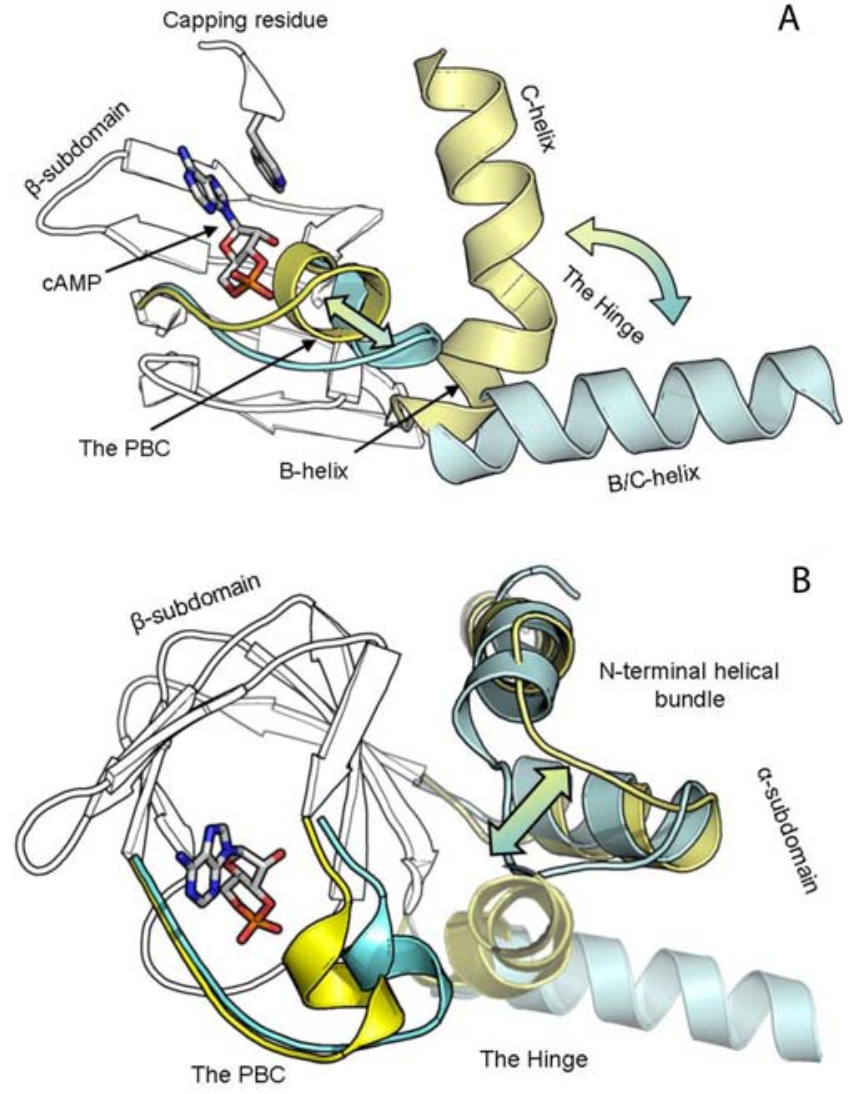

Figure 1. Current understanding of the major CAMP-CNB domain interactions represented by the PKA regulatory subunit type Ia: A-domain. (A) Correlated movement of the PBC and the Hinge. CAMP-bound conformation (B-form) is colored yellow; holoenzyme conformation (H-form) is colored cyan. The cAMP and the capping residue positions are shown. (B) N-terminal part of the $\alpha$ subdomain performs reverse motion (with respect to the Hinge), contacting the $\mathrm{PBC}$ in the $\mathrm{H}$-form.

doi:10.1371/journal.pcbi.1000056.g001

protein structure, and the result of this alignment is a pair of isomorphic graphs. Vertices of the graphs correspond to the residues which form similar spatial patterns in both proteins. Each vertex/residue is connected to the rest of the graph by several edges. They indicate the residue neighbors whose positions and orientation in space are conserved with respect to this residue. As we have shown earlier [15,21], functionally important residues of protein kinases have numerous connections on the similarity graphs. In the previous works we considered only surface exposed residues. Here we analyze all residues. This allows us to recognize conserved motifs that are buried in the protein core. We define a term "involvement score" (IS) of a particular residue, which is equal to the number of edges for the corresponding vertex on the graph provided by the LSP alignment procedure. It reflects the extent of participation of this residue in formation of invariant spatial patterns and corresponds to $\mathrm{AA}$ and $\mathrm{AI}$ scores used in the previous work [21], where we compared active and inactive protein kinases.

Earlier we used the LSP alignment for comparison of different proteins having similar functions. In this work we present an alternative way of using the LSP alignment program. Our purpose was to quantify cAMP-induced structural rearrangements in different CNB domains. This was made by aligning two different conformations of the same protein. As the IS reflects only local structural similarities (in our case within $10 \AA$ range between $\mathrm{C}_{\alpha^{-}}$ 


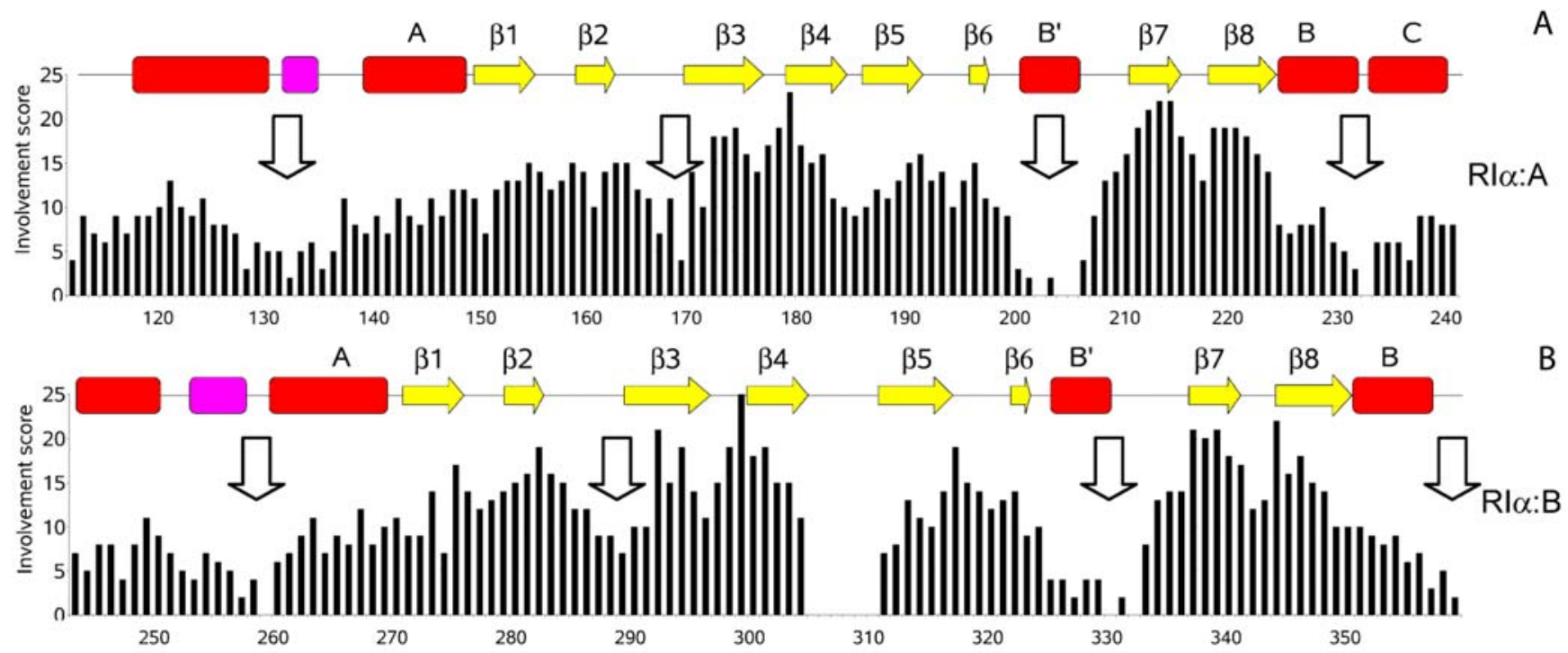

Figure 2. Study of the CAMP-induced conformational changes in PKA:RI $\alpha$ by LSP-alignment. High involvement scores correspond to relatively rigid parts of the molecule. Low values of the score characterize elements, which are the most sensitive to the presence of cAMP. Secondary structure is shown by red rectangles $\left(\alpha\right.$-helix), magenta rectangles $\left(3_{10}\right.$-helix), and yellow arrows ( $\beta$-strands). Four allosteric "hot spots" for each domain are shown by arrows. doi:10.1371/journal.pcbi.1000056.g002
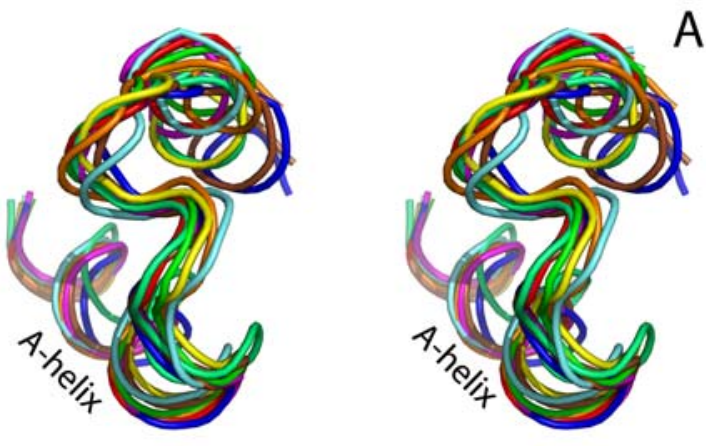

C-subunit
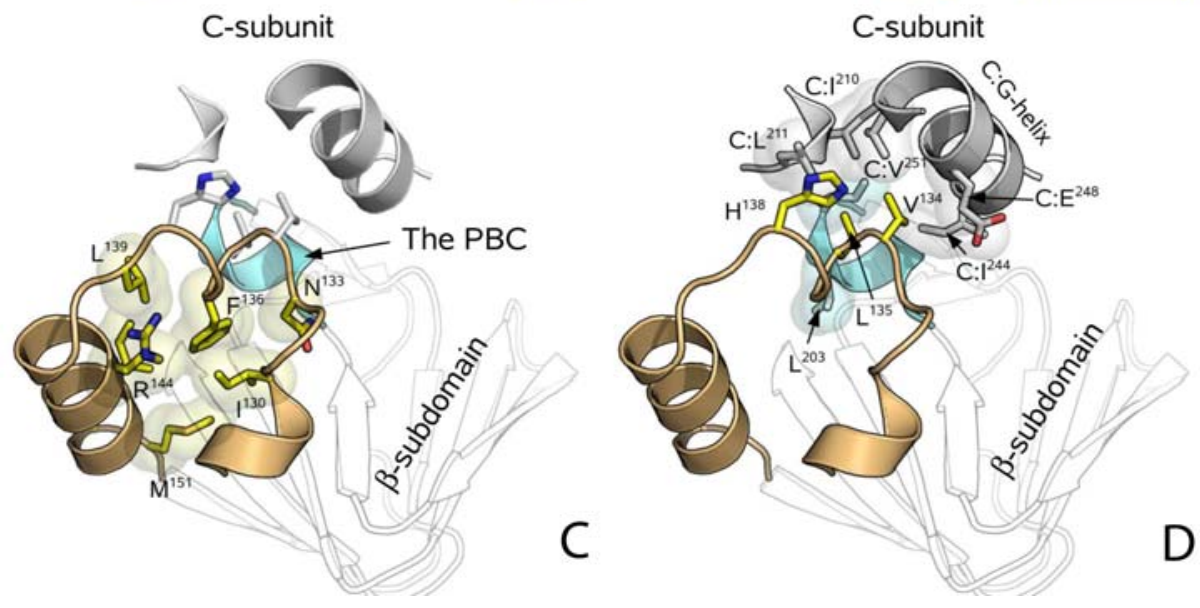

Figure 3. The conserved N3A-motif in the $\mathbf{N}$-terminal part of the $\boldsymbol{\alpha}$-subdomain. (A) Stereo picture of N3A-motifs from 9 different CNB domains: A and B domains of PKA types Rl $\alpha$, RII $\alpha$, and RII $\beta$; Epac2; ionic channel HCN; and potassium channel MloK1. (B) Sequence alignment of the 9 N3A-motifs. $\alpha$-helical regions are shaded magenta. Residues with negative chirality are shaded yellow. Hydrophobic residues or residues with large aliphatic segments are shown in bold. Colored circles correspond to the coloring on the stereo picture. (C) Hydrophobic interactions between residues of the N3A-motif provide integrity of the structural element. N3A-motif of PKA:Rl $\alpha$ A-domain is colored tan. Interacting residues are colored yellow. Connelly surfaces around their aliphatic parts are shown. (D) Residues on the tip of the $3_{10}$-loop are involved in protein-protein interactions in the PKA holoenzyme. The PBC is colored cyan. C-subunit is colored grey.

doi:10.1371/journal.pcbi.1000056.g003 


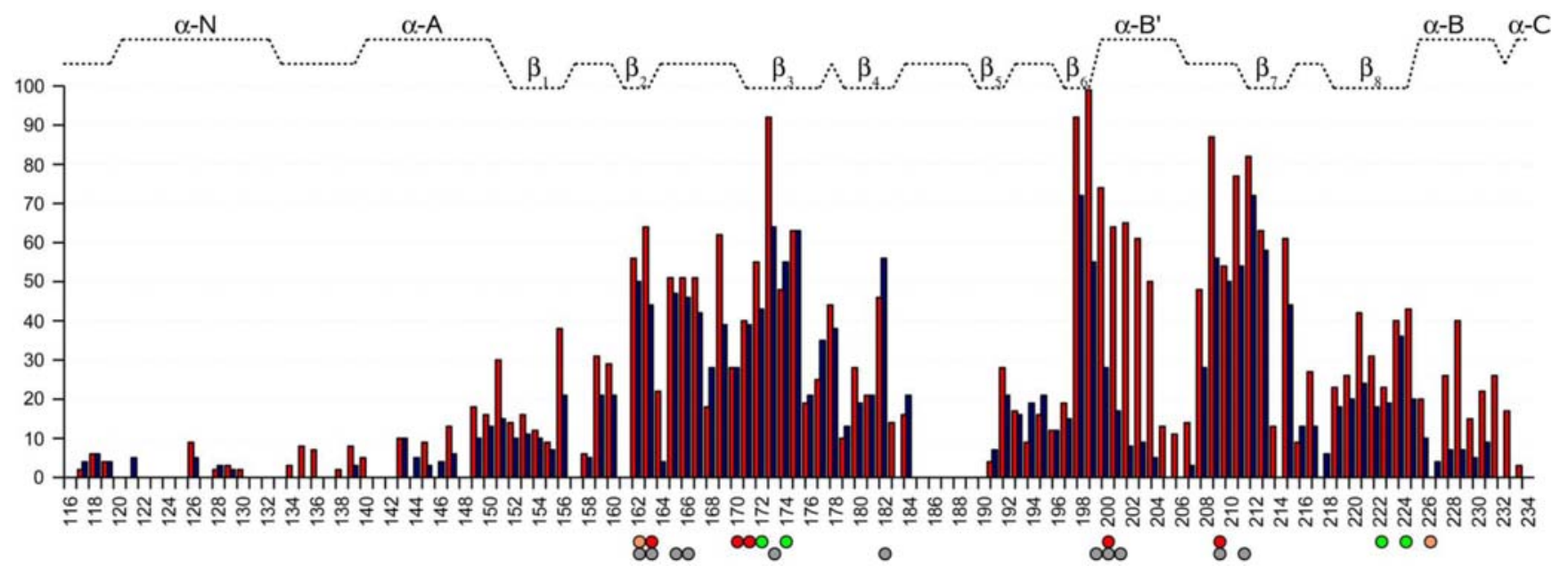

Figure 4. Accumulated involvement scores obtained by LSP alignment different CNB domains. $B$ - form of Rl $\alpha$ : $A$ was compared to $B$ forms of Rl $\alpha: B, R\|\beta: A, R\| \beta: B, H C N$, and MloK1 (red bars); and H-forms of Rl $\alpha: B, R\|l \alpha: A, R\| l \alpha: B, E p a c$, and MloK1 (blue bars). Colored circles indicate the "three-shell" model[16] residues: $1^{\text {st }}$ shell, red; $2^{\text {nd }}$ shell, yellow; and $3^{\text {rd }}$ shell, green. Dark grey circles indicate the residues, which were found to be conserved previously [15].

doi:10.1371/journal.pcbi.1000056.g004

atoms) any large scale rearrangements in the protein do not change the score significantly. Therefore, residues which form rigid structures inside the protein and maintain their relative positions will have a high level of IS. In contrast, those residues located in points of protein flexibility will have low IS, reflecting the loss of similarity between the two protein structures. One can speculate that the residues with the lowest IS can play an important role in the allosteric mechanism, as such elements like "hinges" or "switches" have to accept two distinctive "on" and "off" conformations.
Recognition of a Conserved Novel Allosteric "N3A-Motif"

Figure 2 presents the results of LSP alignment of PKA (RI $\alpha)$ Hand B-forms. As expected, residues from the rigid $\beta$-sandwich had the highest IS values, reflecting the rigidity of the $\beta$ subdomain. In contrast, four regions showed a significant decrease in IS: 1) B'helix of the PBC; 2) the hinge region; 3) the $\beta_{2,3}$-loop and 4) $\mathrm{N}$ terminal part of the $\alpha$-subdomain. These results are in good correspondence with the NMR studies of the RI $\alpha$ A-domain [16]. Our analyses show that both A- and B-domains have similar IS profiles, although the drop in the $\beta_{2,3}$-loop in $\mathrm{B}$-domain was less

\begin{tabular}{|c|c|c|c|c|c|c|c|c|c|}
\hline & \#1 & \#2 & \#3 & \#4 & \#5 & \#6 & \#7 & \#8 & \#9 \\
\hline RI $\alpha: A$ numbering & 163 & 164 & 165 & 166 & 167 & 168 & 169 & 170 & 171 \\
\hline $\mathrm{RI} \alpha: \mathrm{A}$ & ${ }^{R}$ I & D & $\beta_{4,5} Q_{\beta_{3}}$ & $\mathrm{G}_{\mathrm{R}}$ & $D_{R}$ & $E$ & $G_{R}$ & ${ }^{B} D_{\alpha_{g}}$ & $N_{R}$ \\
\hline RII $\alpha: A$ & ${ }^{R}$ I & D. & $\beta_{4,5} Q_{\beta}$ & $\mathrm{G}_{\mathrm{R}}$ & $D_{R}$ & D & $\mathrm{G}_{R}$ & D & $N_{R}$ \\
\hline$R \| \beta: A$ & ${ }^{R}$ I & D & $\beta_{4,5} Q_{\beta}$ & $\mathrm{G}_{\mathrm{R}}$ & $D_{R}$ & $D$ & $\mathrm{G}_{\mathrm{R}}$ & & $\mathrm{N}_{\mathrm{R}}$ \\
\hline $\mathrm{R} \mid \alpha: \mathrm{B}$ & ${ }^{R} V$ & v & $\beta_{4,5} Q_{\beta}$ & $\mathrm{G}_{\mathrm{R}}$ & $E_{R}$ & $P$ & $\mathrm{G}_{\mathrm{R}}$ & $D_{\alpha_{k}}$ & $E_{R}$ \\
\hline$R \| \alpha: B$ & ${ }^{R}$ I & A & $\beta_{4,5} Q_{\beta}$ & $\mathrm{G}_{R}$ & $E_{R}$ & $K$ & ${ }^{R} A$ & $D_{\alpha_{\mathrm{B}}}$ & $S_{R}$ \\
\hline$R \| \beta: B$ & ${ }^{R}$ I & A & $\beta_{4,5} Q_{\beta_{7}}$ & $\mathrm{G}_{\mathrm{R}}$ & $D_{R}$ & $\mathrm{~S}$ & ${ }^{R} A$ & $\mathrm{D}_{\alpha_{\mathrm{s}}}$ & $\mathrm{S}_{\mathrm{R}}$ \\
\hline $\mathrm{HCN}$ & ${ }^{R_{1}}$ & $\mathrm{R}$. & ${ }^{?} E_{\beta_{3}}$ & $G_{R}$ & $\mathrm{~T}_{R}$ & I & $G_{R}$ & $\mathrm{~K}_{\alpha}$ & $K_{R}$ \\
\hline Epac & ${ }^{R} F$ & $\mathrm{~N}$ & $\beta_{4,5} Q_{\beta}$ & $\mathrm{G}_{\mathrm{R}}$ & $E_{R}$ & $E$ & $\mathrm{G}_{\mathrm{R}}$ & $\hat{\mathrm{T}}_{g}$ & $S_{R}$ \\
\hline Mlok1 & ${ }^{\mathrm{R}} \mathrm{C}$ & $\mathrm{R}$. & $I_{\beta}$ & $\mathrm{G}_{R}$ & $E_{R}$ & P & $\mathrm{G}_{\mathrm{R}}$ & $\mathrm{D}_{\alpha_{\mathrm{B}}}$ & $\mathrm{R}_{\mathrm{R}}$ \\
\hline Chirality & - & - & + & + & - & - & + & + & - \\
\hline
\end{tabular}

Figure 5. Sequence alignment of $\boldsymbol{\beta}_{2,3}$-loops for different CNB domains. Contacts formed by the residues are shown either on the upper left side (side chain) or lower right side (main chain). Contacts to the $\mathrm{R}^{209}$ are indicated by capital $\mathrm{R}$ : green, hydrophobic; red, polar. Also contacts to the PBC, B-helix, $\beta_{4,5}$-loop, and $\beta_{7}$-strand are indicated. Question mark signifies that the $\beta_{4,5}$-loop is not resolved in the HCN structure. Dashed arrows show important hydrogen bonds: above the residue letters, between side chains; under the letters, between their main chains. The last row presents main chain chirality sign for the residues.

doi:10.1371/journal.pcbi.1000056.g005 


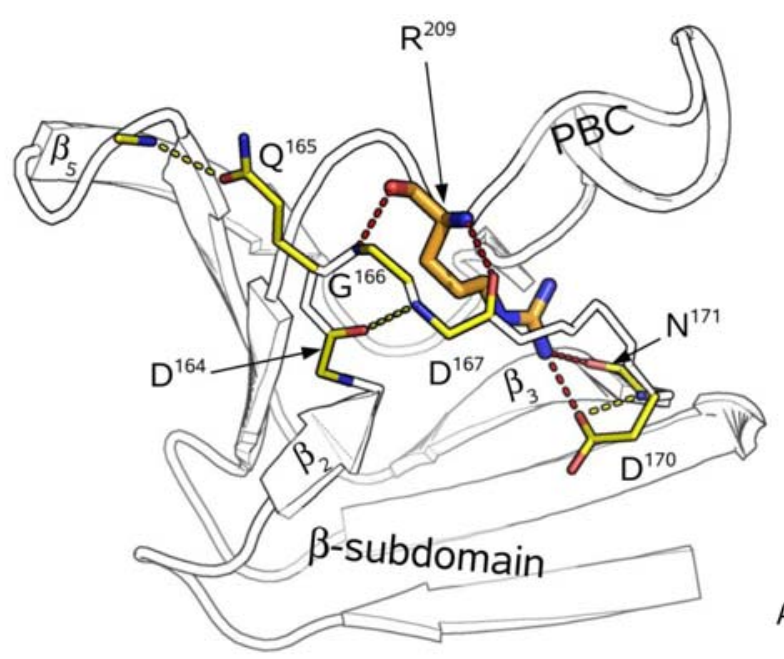

A

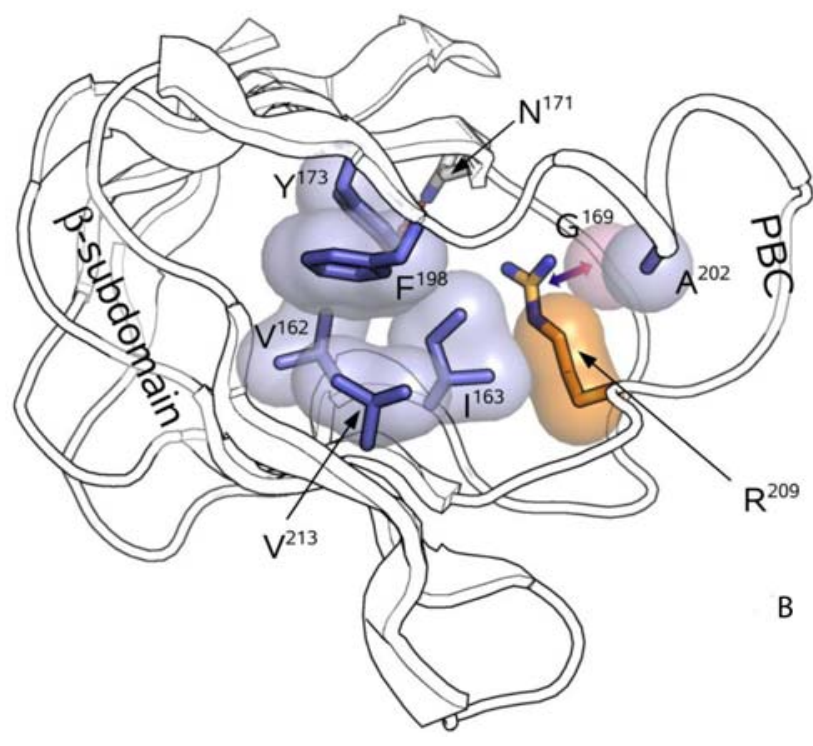

Figure 6. Highly conserved $\beta_{2-3}$-loop secures the PBC-arginine side chain position. (A) Major polar interactions of the $\beta_{2-3}$-loop (RI $\alpha$ :A case). $R^{209}$ is colored tan. Other residues involved in the interactions are colored yellow. (B) Nonpolar polar interactions surrounding the PBC-arginine. Hydrophobic residues are colored blue, and their Connelly surfaces are shown. $\mathrm{CH}-\pi$ interaction between the conserved $\mathrm{G}^{169}$ and $\mathrm{R}^{209}$ is indicated by an arrow.

doi:10.1371/journal.pcbi.1000056.g006

prominent. Similar results were obtained for the A-domain of PKA $(\mathrm{RII} \beta)$ and the potassium channel MloKl (Figure S1). The decrease of IS in the $\beta_{2,3}$-loop in the potassium channel CNB domain was not as striking as in A-domains of PKA (both RI $\alpha$ and RII $\beta$ ), but similar to the B-domain of RI $\alpha$. This is in a good agreement with the earlier observation that $\mathrm{B}$-domains of PKA are more similar to the rest of CNB domains, than the A-domains [15].

The N-terminal helical structure which earlier was called an "N-terminal helical bundle" has not been considered as a conserved element [14]. It is not a part of the current CNB domain nomenclature for two reasons: first - it is not present in CAP, and second - in the different CNB domains it forms slightly different secondary structures. For example in the B-form of RI $\alpha$, RII $\beta$, MloK1 and Epac2 it contains a short $33_{10}$-helix. In the $\mathrm{H}$ form of RI $\alpha$ :A it contains a set of 3-turns, which do not form the classical 310 -helix and is considered to be a loop, while the B-

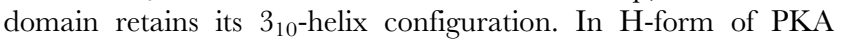

RII $\alpha$ :A and RII $\beta$ :A this element qualifies as an $\alpha$-helix, but in the B-form of RII $\beta$ :A and RII $\beta$ :B it is a $3_{10}$-helix. However, a close look at the middle part of the bundle shows that the geometry of its backbone in different CNB domains (both $\mathrm{H}$ - and B-forms), is rather conserved (Figure 3A). Moreover, this element, which we will define as a " $310^{-}$loop", has a distinctive pattern of phi/psi angles: Figure 3B shows a sequence alignment of different $\mathrm{N}$ terminal helical bundles. One can see that A-helix of the presented CNB domains is preceded by an eight residue long loop. Its first conserved feature is that both ends of the loop contain residues with negative chirality (a characteristic of $\beta$-strands): one at the $\mathrm{N}$ terminus and two at the C-terminus. There is also a large conserved hydrophobic residue (phenylalanine or leucine) in the middle of the loop $\left(\mathrm{F}^{136}\right.$ Figure $\left.3 \mathrm{C}\right)$, which plays a central role in the hydrophobic cluster formed by the A-helix and the preceding $\alpha$-helix, which did not have an established name. As in the PKA$\mathrm{RI} \alpha$ A-domain, it was called " $\alpha \mathrm{X}_{\mathrm{n}}$-helix" [16] or "X:N-helix" [6], here we call it "N-helix", and the combination of the N-helix, the 3 10-loop and A-helix structure - the N3A-motif. The characteristic feature of this motif is the presence of multiple $\mathrm{X}-\mathrm{X}$ pairs in its sequence (where $\mathrm{X}$ represents a hydrophobic residue or a residue with large hydrophobic segment such as arginine or asparagine) (Figure 3B). Such residues are closely positioned on one side of the helix and provide a secure connection between the N3A-motif elements (Figure 3G), a feature similar to the tetratricopeptide repeat [22] or the leucine-zipper [23] motifs.

Analysis of the recently discovered holoenzyme structures of PKA shows that the $3{ }_{10}$-loop residues positioned between the $\mathrm{X}-\mathrm{X}$ pairs are usually involved in important interactions. For example, in RIa:A-domain $\mathrm{V}^{134}, \mathrm{~L}^{135}$ and $\mathrm{H}^{138}$ stack against a large hydrophobic cluster formed by the $\mathrm{C}$-subunit and the PBC (Figure 3D). In the RIa:B-domain $\mathrm{S}^{252}$ forms five hydrogen bonds to the $\mathrm{PBC}$, the $\mathrm{C}$ terminal helical structure of the domain and the $\beta$-sandwich.

The suggested conservation of the N3A-motif in different CNB domains raises a question about the definition of $\mathrm{A}$ and $\mathrm{B}$ domains in PKA-R. Until now, the beginning of the B domain was associated with the first residue in it's a-helix (e.g. $\mathrm{W}^{260}$ in RI $\alpha$ or $\mathrm{V}^{280}$ in RII $\beta$ ). Here we suggest a new boundary between the A and $\mathrm{B}$ domains, which will reflect the conservation of the N3Amotif. It is known that the RI $\alpha-(94-244)$ construct retains its functionality and is capable of both binding to cAMP and regulating PKA [24]. In addition, the C-helix of cAMP-bound $\mathrm{RI} \alpha$ has a kink between $\mathrm{Y}^{244}$ and $\mathrm{E}^{245}$. An identical kink exists in the cAMP-bound RII $\beta$ (between $\mathrm{Y}^{265}$ and $\mathrm{E}^{266}$ ). It seems logical to suggest that this kink indicates the border between the $\mathrm{A}$ and $\mathrm{B}$ domains, therefore defining $\mathrm{E}^{245}$ as the beginning of N3A-motif for the B-domain of RI $\alpha$. Such a definition supports the observation made earlier by Huang and Taylor that RI $\alpha$ "residues 245-260 at the end of cAMP binding domain A are structurally more a part of domain B than domain A" [24].

\section{LSP Alignment of Different CNB Domains Emphasizes the Allosteric Role of the $\beta_{2,3}$-Loop}

After the new definition of CNB domains, we used the LSP alignment to detect residues involved in formation of conserved spatial patterns as we did previously for PKA-C [21]. The Adomain of the $\mathrm{RI} \alpha$ holoenzyme was taken as a reference structure. It was compared to five cAMP-bound CNB domains: RI $\alpha: B$, RII $\beta: A$, RII $\beta: B, H C N$ and MloK1. To detect the regions, which respond to the cAMP presence, we also compared our reference structure to five cAMP-free CNB domains: six domains of PKA-R taken from the corresponding holoenzyme complexes: RI $\alpha: B$, RII $\alpha: A, R I I \alpha: B$; and two apo-structures: Epac and MloK1. Involvement scores were accumulated and presented in Figure 4. 


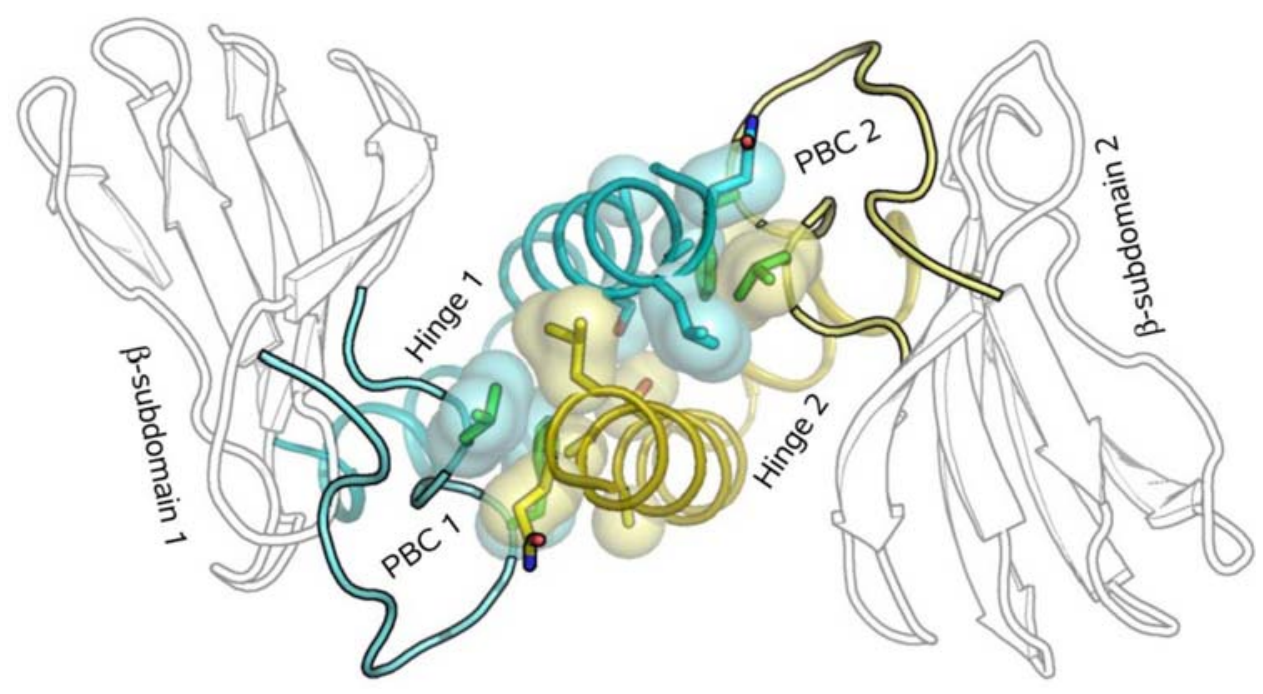

Figure 7. CNB domains of CAP are trans-regulated. Interface between two monomers of CAP is shown (PDB ID, 1CGP). $\beta$-subdomains are shown as black and white contours. The PBC and the Hinge of each monomer are shown as cartoons and colored cyan (first monomer) and yellow (second monomer). Residues which form the hydrophobic interface between PBCs and hinges are shown as sticks with Connelly surfaces. doi:10.1371/journal.pcbi.1000056.g007

The highest involvement scores were detected in the PBC and neighboring $\beta_{6}$ and $\beta_{7}$ strands. This area also had the largest cAMP-induced changes of IS, reflecting the leading role of the $\mathrm{PBC}$ in the allosteric mechanism. Reduced scores in the middle of PBC agree with the sequence variability profiles obtained earlier for PKA-R [25].

The second major region with highly scored residues was in the strands $\beta_{2}, \beta_{3}$ and the loop between them. The result was rather unexpected as, until recently, this area was not considered as an important part of GNB domains. The scores, in general, did not depend on the presence of cAMP, which indicates overall conservation of the loop geometry. This suggests, that the $\beta_{2,3^{-}}$ loop is an important element not only in PKA-R, as it was pointed by Das et. al $[16,26]$, but in all CNB domains. Figure 5 shows a comparison between $\beta_{2,3}$-loops of different CNB domains. As was detected by the DSSP program [27], all loops have the same pattern of their main chain chirality, indicating a high level of their geometry conservation. The most distinctive common feature for all of them is a 3-turn between residues \#2 $\left(\mathrm{D}^{164}\right)$ and \#5 $\left(\mathrm{D}^{167}\right)$ (Figure 6A). It contains an invariant glycine residue \#4 $\left(\mathrm{G}^{166}\right)$ which makes a conserved hydrogen bond to the PBC-arginine carbonyl. The reason for strict conservation of the glycine is evident as its dihedral angles are ruled out for any other type of residue $\left(\phi=89.4^{\circ}\right.$ and $\psi=-26.8^{\circ}$ for $\mathrm{RI} \alpha$ :A). Another conserved hydrogen bond is formed between the PBC-arginine amide and carbonyl of the residue \#5. The third polar contact, which can be found in all CNB structures, is formed between the PBC-arginine guanidinium group and the carbonyl of residue $\# 9\left(\mathrm{~N}^{171}\right)$. The hydrogen bond between the arginine and the side chain of residue $\# 8\left(\mathrm{D}^{170}\right)$, which was found to be important for $\mathrm{RI} \alpha: \mathrm{A}$, is not conserved: it can be seen only in the A domains of RI $\alpha$ and RII $\beta$. This residue, however, often binds to the side chain of residue \#9 and provides communication between the $\beta_{2,3}$-loop and the "hinge" as its carbonyl is always bound to the amide group of the first residue in the B-helix. Besides polar interactions with the PBC-arginine, residue \#1 $\left(\mathrm{I}^{163}\right)$ makes a conserved hydrophobic contact to the arginine side chain. This residue is a member of a conserved hydrophobic core formed by the highly scored residues: $\mathrm{V}^{162}, \mathrm{I}^{163}, \mathrm{Y}^{173}, \mathrm{~F}^{198}$ and $\mathrm{V}^{213}$ (Figure $6 \mathrm{~B}$ ). The important detail is that another member of that cluster: $\mathrm{Y}^{173}$ (one of the three highest scores obtained) - makes a conserved hydrogen bond to the residue $\# 9\left(\mathrm{~N}^{171}\right)$, thus closing the circle around the $\mathrm{PBC}$ arginine.

Glycine is the predominant residue in position \#7, except for the $\mathrm{B}$ domains of RII $\alpha$ and RII $\beta$, where it is substituted by alanine. Any increase of the side chain would lead to a steric clash with highly conserved alanine residues from the $\mathrm{PBC}\left(\mathrm{A}^{202}\right)$, which in its turn also has a hydrophobic contact to the PBC-arginine in the cyclic nucleotide bound configurations.

The hinge, which is a well known element of the allosteric mechanism, demonstrated medium levels of IS and a strong dependence on the presence of cAMP. The preceding $\beta_{8}$-strand received almost the same level of scores. It contains a set of conserved hydrophobic residues, which face the N3A-motif and provide a secure connection of this element to the $\beta$-sandwich. The NMR-study of RI $\alpha$ showed that two residues from the $\beta_{8^{-}}$ strand $\left(\mathrm{W}^{222}\right.$ and $\left.\mathrm{I}^{224}\right)$ had a substantial chemical shift, in response to cAMP binding and are a part of the allosteric mechanism. Our results support this conclusion and demonstrate conservation of the hydrophobic interface through different CNB domains. Another highly scored residue positioned between B-helix and $\beta_{8}$-strand $\left(\mathrm{M}^{151}\right)$ is also a part of this interface.

As we showed earlier the N3A-motifs of different CNB domains have very similar geometry, and conserved sequence motifs. However, this region, except for the C-terminus of A-helix, demonstrated low levels of IS. This indicates that the residues, conserved in the sequence, do not form a rigid spatial motif. This conclusion is supported by the fact that the N-helix and 310 -loop in RI $\alpha$ :A-domain have an elevated level of hydrogen-deuterium exchange $[10,16]$. Apparently, the N3A-motif is a rather flexible element, which can adopt slightly different conformations accommodating the PBC and the hinge movements.

In all comparisons the loop between $\beta_{4}$ and $\beta_{5}$-strands received zero level of IS (Figure 4). It is consistent with the fact that this part of the CNB domain is the least conserved in terms of sequence and structure [28]. Our data show that in many CNB domains the N-terminus of the $\beta_{4-5}$-loop involved in the conserved anchoring of the $\beta_{2-3}$-loop via its \#3 residue $\left(\mathrm{Q}^{165}\right)$ (Figures 5 and $\left.6 \mathrm{~A}\right)$. The $\beta_{4-5^{-}}$ loop spatially comes close to the $\mathrm{C}$-terminus of the $\mathrm{CNB}$ domain and in the RI $\alpha$ :B-domain may provide a docking site for another protein. 


\section{CNB Domains in CAP Are trans-Regulated}

We deliberately excluded the CAP CNB domain from the current analysis as it does not contain the classic N3A-motif. There are several distinctive features that distinguish the CAP CNB domain from those discussed above. The major difference is that in functionally active CAP the two identical CNB domains form a homodimer with the interface being formed mainly by their $\mathrm{C}$ helices. Regulatory subunits of PKA also contain two CNB domains, but their mutual interaction is rather limited: e.g. A-helix of B-domain contacts cAMP and the hinge from the A-domain. In contrast, in CAP the interaction between the two monomers is the most important allosteric contact between the PBC and the hinge. Figure 7 shows that the major binding partner for each PBC is not the hinge from its own $\mathrm{CNB}$ domain but the hinge from the opposite monomer. Such interactions separate the CAP CNB domain from the other CNB domains studied in this work, which we propose to call cis-regulated CNB domains while defining CAP CNB domain as a trans-regulated CNB domain.

\section{Discussion}

The CNB domain, highly conserved throughout biology, is comprised of a set of motifs that both define the docking site for cAMP as well as an allosteric switch that allows it to assume a dramatically different structure when binding to another protein element. Using our new computational method we have analyzed both the allosteric mechanism and the structural motifs conserved in different CNB domains. LSP-alignment proved to be an effective tool for detection of the allosteric "hot spots" - residues with the most significant rearrangement of their side chains. The comparison of $\mathrm{H}$ - and $\mathrm{B}$-forms of PKA-RI $\alpha$ pointed to four major elements of the allosteric mechanism: the $\mathrm{PBC}$, the hinge, the $\beta_{2,3^{-}}$ loop and the N-terminal helical structure. Based on our analysis the latter is now recognized as a new conserved element of cisregulated $\mathrm{CNB}$ domains - the N3A-motif. It is not present in at least one trans-regulated CNB domain - CAP; however, it can be found in another transcriptional regulator, Crp/Fnr family from Porphyromonas gingivalis [29] (PDB ID: 2GAU). The possible role of the N3A-motif in trans-regulated CNB domains needs to be elucidated, but the fact that the allosteric interface between $\alpha$ and $\beta$-subdomains is significantly different (Figure 7) indicates a possible difference in allosteric mechanisms.

The detection of four allosteric hot spots in PKA is consistent with NMR studies of the RI $\beta$ :A-domain [16]. Our analysis shows that the N3A-motif contains two types of residues: hydrophobic $\mathrm{X}-\mathrm{X}$ repeats, which provide structural stability to the element as well as its interactions within the CNB domain (Figure 3B and 3C). The other set of residues is positioned on the tip of the $33_{10}$-loop and is often involved in functionally important protein-protein interactions (Figure 3D).

The results of different cis-regulated CNB domains LSPalignment were in good correspondence with our earlier analysis of CNB domain surface: all eleven residues that were found to be conserved [15], received very high IS values. Our results also support the proposed the "three shells" model of allostery [16]: high levels of IS were detected in the sites of all three shells localization: $\beta_{2}, \beta_{3}$ and $\beta_{8}$ strands (Figure 4).

The most unexpected result was a very high scoring in the $\beta_{2,3^{-}}$ loop region, which was not included previously in the general model of CNB domain allostery [14]. This brings up a question of the general role of the $\beta_{2,3}$-loop in different CNB domains. As we showed, its residues always participate in multiple, highly conserved, polar and hydrophobic interactions with the PBC arginine $\left(\mathrm{R}^{209}\right.$ Figure 6$)$. The guanidinium group of this residue makes a very important hydrogen bond to the equatorial oxygen of the cAMP ribose phosphate. Perturbation of the bond, with an arginine-to-lysine mutation [30], or with substitution of the oxygen by sulfur [31], significantly disrupts the cAMP-related allostery. A close look at the $\beta_{2,3}$-loop interactions with the PBC arginine,
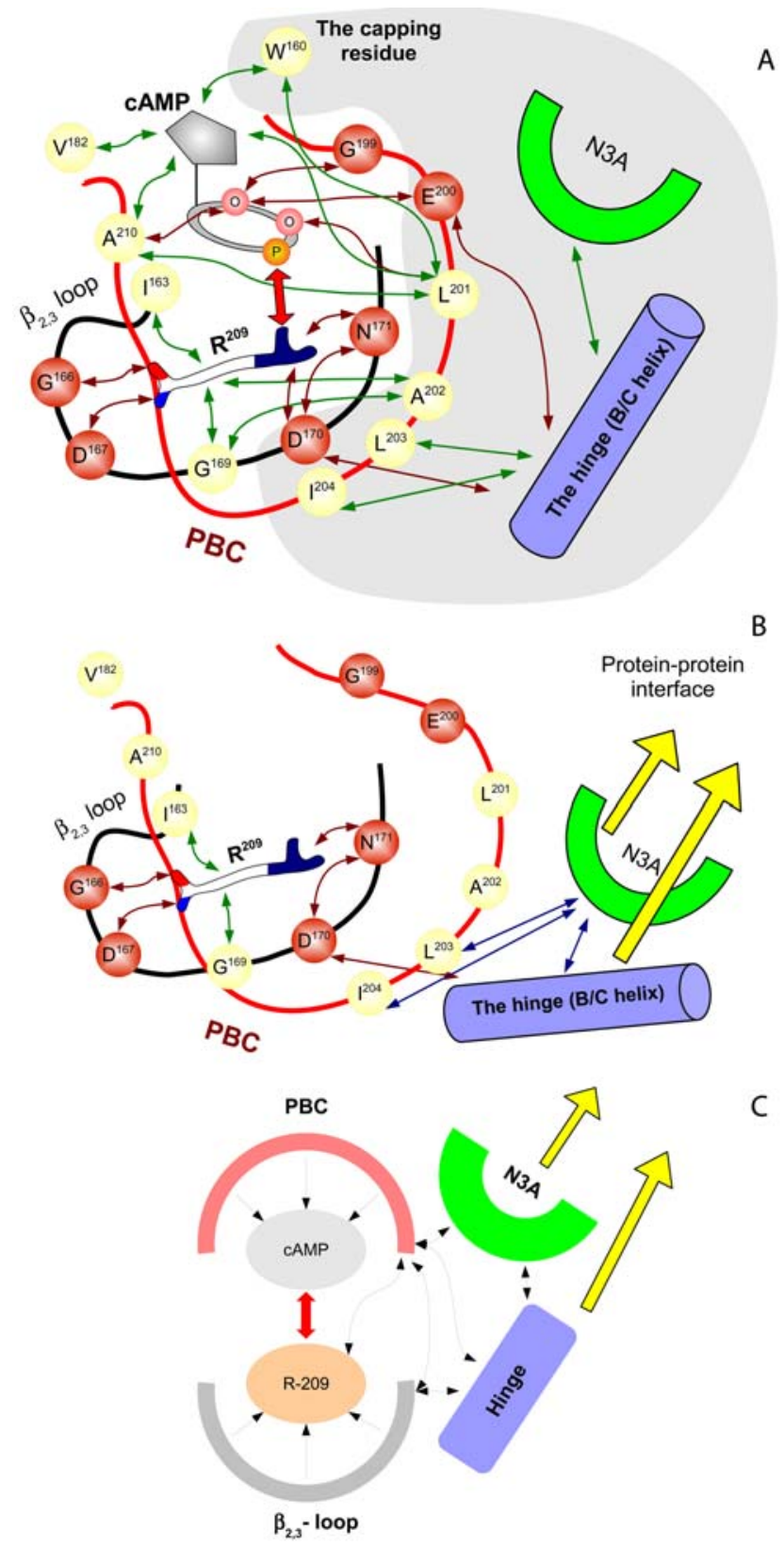

Figure 8. General allosteric mechanism for different CNB domains (RIa:A case). (A) Major interactions between CAMP, the PBC (red) and the $\beta_{2,3}$-loop (black) in cAMP-bound state. Red circles represent residues forming polar bonds (red arrows); yellow circles show residues making hydrophobic contacts (green arrows). The most important bond between CAMP and $R^{209}$ is shown by a double red arrow. Residues and structure elements changing their positions upon CAMP binding are shaded grey. (B) CAMP-free configuration. $\mathrm{R}^{209}$ becomes much less restricted. (C) General diagram of major interactions in the CNB domain. The PBC controls CAMP, the $3_{10}$-loop controls $\mathrm{R}^{209}$, and their interaction provides correct orientation of the hinge region and the N3A motif, which form a protein-protein interface. doi:10.1371/journal.pcbi.1000056.g008 
shows that it "surrounds" the residue, immobilizing its bulky side chain. The position and total geometry of the loop is remarkably conserved in all CNB domains. The central element of the loop is a universally conserved 3-turn between the positions \#2 $\left(\mathrm{D}^{164}\right)$ and $\# 5\left(\mathrm{D}^{167}\right)$. Spatial orientation of the turn is secured by another conserved interaction of residue $\# 3\left(Q^{165}\right)$ with the $\beta_{4,5^{-}}$ loop. The apparent reason for such rigidity is a correct positioning of the PBC arginine main chain, which is locked by two hydrogen bonds to residues $\# 4\left(\mathrm{G}^{166}\right)$ and \#5 $\left(\mathrm{D}^{167}\right)$. The tip of the arginine side chain is always bound to the \#9 residue main chain $\left(\mathrm{N}^{171}\right)$. Adomains of PKA regulatory subunits RI $\beta$ and RII $\beta$ have an additional hydrogen bond to the side chain of residue $\# 8\left(\mathrm{D}^{170}\right)$. This interaction, however, is not conserved in other CNB domains. Alternatively, a polar bond between the eighth residue main chain and N-terminus of the B-helix was found in all studied structures. Thus, the possible role of the residue is to communicate between the PBC arginine and the hinge. The direct binding of residue \#8 to the arginine, which is present in the PKA-R Adomains can substantially reinforce such communication.

These finding lead us to a suggestion that immobilization of the PBC arginine side chain has to be an important feature of the allosteric mechanism. Definitely, without a stable guanidinium group, one hardly can expect a stable bond with the ribose phosphate moiety of cAMP. Here we propose a general model describing the cyclic nucleotide related allostery (Figure 8). The model is built around interactions between the cyclic nucleotide phosphate and the PBC-arginine guanidinium group (red arrow in Figure 8G). The main suggestion of the model is that the guanidinium group is very mobile and can not form a stable bond unless it is restricted by numerous interactions of the arginine with the $\mathrm{PBC}$ and $\beta_{2,3}$-loop. This model is supported by the observation that the PBC-arginine side chain is not totally buried in the CNB domain, but partially exposed to the outer solution. According to the DSSP calculation [27], its water accessible area in the cAMPfree structures vary between $16 \AA^{2}$ in the potassium channel to $30 \AA^{2}$ in $\mathrm{RI} \alpha$ :A.

According to the proposed model stable cyclic nucleotide binding can be achieved only by interaction between all the major elements of the CNB domain: the PBC, the PBC-arginine, $\beta_{2,3^{-}}$ loop, the hinge and the N3A-motif. Almost all elements interact with each other, leading to a rather complex allosteric mechanism. The primary function of the $\beta_{2,3}$-loop is to position all parts of the arginine including the backbone, the hydrophobic part of the side chain and the guanidinium moiety. It also interacts with the hinge (through the residue \#8) and the PBC (via hydrophobic contact between residue $\# 7$ and $\mathrm{A}^{202}$ ). The PBC serves, first of all, as a cyclic nucleotide molecule stabilizer, but it also plays an important role in immobilization of the arginine side chain (via $\mathrm{A}^{202}$ ). In addition, it interacts with the hinge and the N3A-motif, which usually forms protein-protein interface, providing the transition of

\section{References}

1. Beavo JA, Brunton LL (2002) Cyclic nucleotide research - still expanding after half a century. Nat Rev Mol Cell Biol 3: 710-718.

2. Bos JL (2003) Epac: a new cAMP target and new avenues in cAMP research. Nat Rev Mol Cell Biol 4: 733-738.

3. Kaupp UB, Seifert R (2002) Cyclic nucleotide-gated ion channels. Physiol Rev 82: 769-824.

4. Kopperud R, Krakstad C, Selheim F, Doskeland SO (2003) cAMP effector mechanisms. Novel twists for an 'old' signaling system. FEBS Lett 546: 121-126.

5. Newton RP, Smith CJ (2004) Cyclic nucleotides. Phytochemistry 65: 2423-2437.

6. Kim C, Xuong NH, Taylor SS (2005) Crystal structure of a complex between the catalytic and regulatory (RIalpha) subunits of PKA. Science 307: 690-696.

7. Dostmann WR (1995) (RP)-cAMPS inhibits the cAMP-dependent protein kinase by blocking the cAMP-induced conformational transition. FEBS Lett 375: 231-234. the allosteric signal to the molecule, which contains the CNB domain.

\section{Conclusions}

The LSP-alignment of $\mathrm{H}$ - and B-forms of different $\mathrm{CNB}$ domains revealed four conserved structural motifs: the PBC, the Hinge, the N3A-motif and the $\beta_{2,3}$-loop. These elements were found in all studied cis-regulated CNB domains. The N3A motif is not present in CAP, which represents a trans-regulated GNB domain family. We propose a generalized allosteric mechanism for cis-regulated domains as follows: a) The PBC is a primary element, which binds sugar-phosphate moiety of cAMP. b) The $\beta_{2,3}$-loop regulates the cAMP binding to the $\mathrm{PBC}$ via the conserved $\mathrm{PBC}$ arginine. c) Both the $\mathrm{PBC}$ and the $\beta_{2,3}$-loop communicate with the Hinge, which transfers the allosteric signal further to the N3A motif. d) The N3A-motif is the most malleable element of a CNB domain as it provides communication to the host protein.

\section{Methods}

The following structures were used in the current work: PKA:RI $\alpha$ B-form [32] (PDBID - 1RGS); PKA:RI $\alpha$ H-form [8]; PKA:RII $\alpha$ H-form [20]; PKA:RII $\beta$ B-form [13] (1CX4); PKA:RII $\beta$ H-form (Brown et al., unpublished results); HCN B-form [33] (1Q43); MloK1 B-form [19] (1VP6); MloK1 H-form [19] (1U12); Epac2 H-form [34] (2BYV). LSP-alignment was made by previously reported algorithm for surface matching [21]. All residues (both water accessible and buried inside protein) were included in the analysis. For that reason, the water accessibility cut-off was taken equal to zero. Residues were represented by their $\mathrm{C}_{\alpha}-\mathrm{C} \beta$ vectors. The maximum distance between $\mathrm{C}_{\alpha}$ atoms was $12 \AA$. Tolerance for $\mathrm{C}_{\alpha}-\mathrm{C}_{\alpha}$ distance was $0.4 \AA$. Tolerance for $\mathrm{C}_{\alpha}-$ C $\beta$ distance was $0.75 \AA$. Tolerance for the dihedral angle between the vectors was $30^{\circ}$. Residues with the BLOSUM62 score greater than or equal to 1 were considered to be similar. Calculations were made on a personal computer (Pentium 4; $1.8 \mathrm{GHz}$; Gb RAM) under Linux OS. Molecular graphics were prepared using PyMOL (DeLano Scientific, San Carlos, CA).

\section{Supporting Information}

Figure S1 Study of the cAMP-induced conformational changes. Changes in (A) PKA:RII $\beta$ (A-domain) and (B) potassium channel (MloK1) by LSP-alignment.

Found at: doi:10.1371/journal.pcbi.1000056.s001 (0.09 MB DOG)

\section{Author Contributions}

Conceived and designed the experiments: AK. Performed the experiments: AK. Analyzed the data: AK ST LT. Wrote the paper: AK ST.

8. Kim C, Cheng CY, Saldanha SA, Taylor SS (2007) PKA-I holoenzyme structure reveals a mechanism for cAMP-dependent activation. Cell 130: $1032-1043$.

9. Wu J, Brown SH, von Daake S, Taylor SS (2007) PKA type IIalpha holoenzyme reveals a combinatorial strategy for isoform diversity. Science 318: 274-279.

10. Anand GS, Hughes CA, Jones JM, Taylor SS, Komives EA (2002) Amide H/2H exchange reveals communication between the cAMP and catalytic subunitbinding sites in the $\mathrm{R}(\mathrm{I})$ alpha subunit of protein kinase A. J Mol Biol 323: 377-386.

11. Li F, Gangal M, Jones JM, Deich J, Lovett KE, et al. (2000) Consequences of cAMP and catalytic-subunit binding on the flexibility of the A-kinase regulatory subunit. Biochemistry 39: 15626-15632.

12. Wu J, Brown S, Xuong NH, Taylor SS (2004) RIalpha subunit of PKA: a cAMP-free structure reveals a hydrophobic capping mechanism for docking cAMP into site B. Structure 12: 1057-1065. 
13. Diller TC, Madhusudan, Xuong NH, Taylor SS (2001) Molecular basis for regulatory subunit diversity in cAMP-dependent protein kinase: crystal structure of the type II beta regulatory subunit. Structure 9: 73-82.

14. Rehmann H, Wittinghofer A, Bos JL (2007) Capturing cyclic nucleotides in action: snapshots from crystallographic studies. Nat Rev Mol Cell Biol 8: 63-73.

15. Berman HM, Ten Eyck LF, Goodsell DS, Haste NM, Kornev A, et al. (2005) The cAMP binding domain: an ancient signaling module. Proc Natl Acad Sci U S A 102: 45-50.

16. Das R, Esposito V, Abu-Abed M, Anand GS, Taylor SS, et al. (2007) cAMP activation of PKA defines an ancient signaling mechanism. Proc Natl Acad Sci U S A 104: 93-98.

17. Gibson RM, Ji-Buechler Y, Taylor SS (1997) Interaction of the regulatory and catalytic subunits of cAMP-dependent protein kinase. Electrostatic sites on the type Ialpha regulatory subunit. J Biol Chem 272: 16343-16350.

18. Zawadzki KM, Hamuro Y, Kim JS, Garrod S, Stranz DD, et al. (2003) Dissecting interdomain communication within cAPK regulatory subunit type IIbeta using enhanced amide hydrogen/deuterium exchange mass spectrometry (DXMS). Protein Sci 12: 1980-1990.

19. Clayton GM, Silverman WR, Heginbotham L, Morais-Cabral JH (2004) Structural basis of ligand activation in a cyclic nucleotide regulated potassium channel. Cell 119: 615-627.

20. Wu J, Brown S, von Daake S, Taylor SS (2007) PKA type IIa holoenzyme reveals a combinatorial strategy for isoform diversity. Science 318: 274-279.

21. Kornev AP, Haste NM, Taylor SS, Ten Eyck LF (2006) Surface comparison of active and inactive protein kinases identifies a conserved activation mechanism. Proc Natl Acad Sci U S A 103: 17783-17788.

22. Blatch GL, Lassle M (1999) The tetratricopeptide repeat: a structural motif mediating protein-protein interactions. Bioessays 21: 932-939.

23. Lupas A (1996) Coiled coils: new structures and new functions. Trends Biochem Sci 21: 375-382
24. Huang LJ, Taylor SS (1998) Dissecting cAMP binding domain A in the RIalpha subunit of cAMP-dependent protein kinase. Distinct subsites for recognition of cAMP and the catalytic subunit. J Biol Chem 273: 26739-26746.

25. Canaves JM, Taylor SS (2002) Classification and phylogenetic analysis of the cAMP-dependent protein kinase regulatory subunit family. J Mol Evol 54: $17-29$.

26. Das R, Melacini G (2007) A model for agonism and antagonism in an ancient and ubiquitous cAMP-binding domain. J Biol Chem 282: 581-593.

27. Kabsch W, Sander C (1983) Dictionary of protein secondary structure: pattern recognition of hydrogen-bonded and geometrical features. Biopolymers 22: 2577-2637.

28. Johnson DA, Akamine P, Radzio-Andzelm E, Madhusudan M, Taylor SS (2001) Dynamics of cAMP-dependent protein kinase. Chem Rev 101: 2243-2270.

29. Rotella FJ, Zhang RG, Mulligan R, Moy S, Joachimiak A (2008) The 1.9-A crystal structure of transcriptional regulator, Crp/Fnr family from Porphyromonas gingivalis. In press.

30. Herberg FW, Taylor SS, Dostmann WR (1996) Active site mutations define the pathway for the cooperative activation of cAMP-dependent protein kinase. Biochemistry 35: 2934-2942.

31. Dostmann WR, Taylor SS, Genieser HG, Jastorff B, Doskeland SO, et al. (1990) Probing the cyclic nucleotide binding sites of cAMP-dependent protein kinases I and II with analogs of adenosine $3^{\prime}, 5^{\prime}$-cyclic phosphorothioates. J Biol Chem 265: 10484-10491.

32. Su Y, Dostmann WR, Herberg FW, Durick K, Xuong NH, et al. (1995) Regulatory subunit of protein kinase A: structure of deletion mutant with cAMP binding domains. Science 269: 807-813.

33. Zagotta WN, Olivier NB, Black KD, Young EC, Olson R, et al. (2003) Structural basis for modulation and agonist specificity of HCN pacemaker channels. Nature 425: 200-205.

34. Rehmann H, Das J, Knipscheer P, Wittinghofer A, Bos JL (2006) Structure of the cyclic-AMP-responsive exchange factor Epac2 in its auto-inhibited state. Nature 439: 625-628. 